\title{
Perinatal Outcome of Discordant Anomalous Twins: A Single-Center Experience in a Developing Country
}

\author{
Tatiana R. M. M. Fernandes, ${ }^{1}$ Paulo R. N. Carvalho, ${ }^{1}$ Fernanda B. Flosi, ${ }^{2}$ Ana Elisa R. Baião, ${ }^{1}$ and \\ Sant Claire G. Junior ${ }^{3}$ \\ ${ }^{1}$ Department of Obstetrics, Fernandes Figueira Institute, Rio de Janeiro, Brazil \\ ${ }^{2}$ Perinatal Clinic, Fernandes Figueira Institute, Rio de Janeiro, Brazil \\ ${ }^{3}$ Clinical Research Department, Fernandes Figueira Institute, Rio de Janeiro, Brazil
}

\begin{abstract}
A dramatic increase in twin pregnancies has been observed in the past few decades, primarily related to assisted reproductive techniques (ART) and increased average maternal age during pregnancy. Multiple pregnancies, compared to singleton pregnancies, are associated with greater perinatal morbidity and mortality. The present study evaluated the perinatal outcomes of pregnancies with discordant anomalous twins in a tertiary maternity ward in a developing country. Data were retrospectively collected from the Instituto Fernandes Figueira/FIOCRUZ, Brazil between January 2002 and December 2014. We identified 74 twin pregnancies with discordant anomalous twins. Final data analysis was based on 40 pregnancies. Congenital defects were classified according to the International Classification of Diseases: ICD-10: the digestive system was responsible in $27(34 \%)$ cases; the central nervous system was responsible in $18(22 \%)$ cases; the urinary tract was responsible in $14(17 \%)$ cases; and the circulatory system was responsible in 14 $(17 \%)$ cases. A total of 19 deaths occurred during the study period, and delivery before 30.4 weeks was a significant prediction of fetal death $(p=.01)$. The presence of hydrops in the affected fetus was related to a higher number of deaths in healthy fetuses and contributed to a worse prognosis. The presence of this condition was the cause of $12(55.6 \%)$ deaths in healthy fetuses. A 10 times higher risk of death of a normal co-twin was observed in cases of death of the anomalous twin ( $p=.002$, OR $10.55,95 \% \mathrm{Cl}$ : 1.9-58.52).
\end{abstract}

Keywords: twin pregnancy, malformation, perinatal outcomes, stillbirth

A dramatic increase in twin pregnancies has been observed in the past decades, primarily related to assisted reproductive techniques (ART) and increased average maternal age during pregnancy (American Congress of Obstetricians and Gynecologists [ACOG], 2004).

There were 57,956 twin pregnancies in Brazil in 2013 (DATA SUS, 2015). An increase of 76\% of multiples pregnancy births was observed in the state of Rio de Janeiro between 1990 and 2009 (DATASUS, 2015). This increase is greater than the observed increase in developed countries. For example, the 2011 twin birth rate in the United States was 33.2 per 1,000 total births, and this rate was essentially unchanged from 2009 and 2010. Increases averaged nearly $3 \%$ annually from 1980 to 2004 (peaking at more than $4 \%$ from 1995 to 1998), but the pace of the increase slowed to one half of 1\% annually from 2005 to 2011 (Martin et al., 2012). The multiple maternity rate in the United Kingdom decreased slightly to 15.6 per 1,000 women giving birth in 2013 compared with 15.9 in 2012 (Office for National Statistics, 2014).

Twin gestations are at increased risk for preterm delivery, intrauterine growth restriction, and congenital anomalies (ACOG, 2004; Creasy et al., 2009; Little \& Nevin, 1989b). The presence of malformation in one twin has an impact on the perinatal outcomes of the affected twin and the normal fetus. The incidence of structural defects is approximately 1.5 times greater than singleton pregnancies for all twins. This rate reaches 2.3 when monochorionic (MC)

RECEIVED 9 September 2015; ACCEPTED 6 March 2016. First published online 20 June 2016.

ADDRESS FOR CORRESPONDENCE: Tatiana Romaguera Mathias Marques Fernandes, Department of Obstetrics, Instituto Fernandes Figueira, Rio de Janeiro, Brazil. E-mail: tatiroma1@yahoo.com.br 
twins are compared with dichorionic (DC) twins (Purisch et al., 2008).

Structural anomalies are responsible for the increased risk of preterm delivery, perinatal death, and intrauterine growth restriction in singleton gestation (Purisch et al., 2008; RCOG, 2008). There is an exceptional and challenging obstetric condition among twins that is described as discordant malformation, which occurs when one fetus is apparently normal and the co-twin carries a malformation that is identified using antenatal ultrasound. (Diana et al., 2010; Lust et al., 2008).

The management of discordant malformation is even more complex in some developing countries, where the law does not allow selective feticide (SF) for the abnormal twin even under conditions that are not compatible with neonatal life. The multidisciplinary attending teams in these countries should be aware of the natural history of discordant malformation in twins. The importance of the timing of delivery should be emphasized, considering the balance between intrauterine death and preterm birth (Shek et al., 2014).

The present study investigated perinatal outcomes of discordant anomalous twins in a Brazilian tertiary center where $\mathrm{SF}$ is not performed.

\section{Methods}

Data were collected retrospectively from the Instituto Fernandes Figueira/FIOCRUZ (IFF/FIOCRUZ), Brazil between January 2002 and December 2014. IFF/FIOCRUZ is a tertiary maternity facility in the state of Rio de Janeiro, Brazil.

The following inclusion criteria were used: discordant anomalous malformation diagnosed or confirmed on ultrasound performed at IFF/FIOCRUZ, prenatal care and delivery performed at the same center, and confirmation of the malformation during postnatal care. All ultrasound scans were performed by senior members of the Department of Obstetrics, who are specialized in maternal-fetal medicine.

The following exclusion criteria were used: complications related to childbirth described in the medical records that may have influenced perinatal outcome, lack of information on delivery conditions or neonatal care in the records, no after birth confirmation of a malformation that was previously diagnosed during the prenatal period. $\mathrm{Zy}-$ gosity was not determined in the present study.

Congenital defects were classified according to the 10th revision of the International Statistical Classification of Diseases and Related Health Problems (ICD-10; WHO, 2015).

Adverse outcomes were defined as prematurity, mortality, or the development of the following diagnoses: (1) periventricular-intraventricular hemorrhage; (2) retinopathy; (3) periventricular leukomalacia; (4) mechanical ventilation; (5) necrotizing enterocolitis; or (6) surfactant use
TABLE 1

Maternal Characteristics and Pregnancy Information of Twins With Discordant Malformations

\begin{tabular}{ll}
\hline Characteristics & $\begin{array}{l}\text { Median (range or } \\
\text { percentage or number) }\end{array}$ \\
\hline Maternal age (years) & $25.5(15-41)$ \\
Pregnancies ages in admission (days) & $185(68-185)$ \\
Gestational age at diagnostic (days) & $165(84-233)$ \\
Antenatal betametasone & $13(65 \%)$ \\
Gestational age at delivery (days) & $244(133-280)$ \\
Chorionicity & $50 \%(20)$ \\
$\quad$ DIDI & $37.5 \%(15)$ \\
$\quad$ MONO/DI & $12.5(5)$ \\
\hline MONO/MONO &
\end{tabular}

Note: $\mathrm{DIDI}=$ dichorionic; $\mathrm{MONO} / \mathrm{DI}=$ monochorionic/diamniotic; $\mathrm{MONO} / \mathrm{MONO}=$ monochorionic/monoamniotic.

\section{TABLE 2}

Normal Twin Adverse Outcomes

\begin{tabular}{ll}
\hline Characteristics & Number (percentage) \\
\hline Death & $9(22.5 \%)$ \\
Small for gestational age & $9(22.5 \%)$ \\
Periventricular-intraventricular hemorrhage & $3(7.5 \%)$ \\
Use of surfactant & $3(7.5 \%)$ \\
Mechanical ventilation & $3(7.5 \%)$ \\
Necrotizing enterocolitis & 0 \\
Retinopathy & 0 \\
\hline
\end{tabular}

during the hospital stay. Statistical analyses were performed in SPSS Software version 2.0 using chi-square tests and independent samples test. The significance level was set at 0.05 .

The Institutional Ethics Review Board approved and registered the study protocol (CAAE 0085.0.008.000-11).

\section{Results}

The database search identified 74 twin pregnancies with discordant malformation. The final data analysis was based on 40 pregnancies. Thirty-four cases were excluded because of complications related to childbirth that may have influenced perinatal outcome, a lack of information on delivery conditions or neonatal care in the records, and no afterbirth confirmation of a malformation that was previously diagnosed during the prenatal period.

The mode of delivery was vaginal in $9(22.5 \%)$ cases and cesarean section in $31(77.5 \%)$ cases. Table 1 summarizes the maternal-fetal characteristics and Table 2 summarizes normal twin adverse outcome characteristics.

The commitment of organs and systems, according to the ICD-10 classification, was divided as follows: digestive system $27.2(34 \%)$, central nervous system $17.6(22 \%)$, urinary tract system $13(17 \%)$, circulatory system $13.6(17 \%)$, others $12(15 \%)$, respiratory system $9.6(12 \%)$, eyes, ears, face $5.6(7 \%)$, genital $1.6(2 \%)$, and chromosomal anomalies $1.6(2 \%)$. Table 3 shows the congenital anomalous defects according to the ICD- 10 . 


\section{TABLE 3}

\begin{tabular}{lc} 
Congenital Defects According to the ICD-10 \\
\hline ICD10 & Number (percentage) \\
\hline Central nervous system & $17.6(22 \%)$ \\
Eyes, ears, face & $5.6(7 \%)$ \\
Circulatory system & $13.6(17 \%)$ \\
Respiratory system & $9.6(12 \%)$ \\
Digestive system & $27.2(34 \%)$ \\
Genital & $1.6(2 \%)$ \\
Urinary tract & $13.6(17 \%)$ \\
Musculoskeletal & $9.6(12 \%)$ \\
Chromosomal anomalies & $1.6(2 \%)$ \\
Others & $12(15 \%)$ \\
\hline
\end{tabular}

\section{TABLE 4}

Length of Stay in the Neonatal Intensive Care Unit/Gestational Age at Delivery-Normal Twin

\begin{tabular}{lcl}
\hline ICD-10 & Days (median) & Weeks (mean) \\
\hline Central nervous system & 4.5 & 34.2 \\
Eyes, ears, face & 0 & 33.5 \\
Circulatory system & 10 & 33.8 \\
Respiratory system & 7.8 & 34.0 \\
Digestive system & 7.9 & 34.2 \\
Genital & 0 & 35.2 \\
Urinary tract & 4.1 & 31.5 \\
Musculoskeletal & 3.7 & 27.9 \\
Others & 14.5 & 35.1 \\
Chromosomal anomalies & 0 & 0 \\
\hline
\end{tabular}

\section{TABLE 5}

Death: Normal Twin

\begin{tabular}{lc}
\hline ICD-10 & Percentage \\
\hline Central nervous system & 11.1 \\
Eyes, ears, face & 22.2 \\
Circulatory system & 11.1 \\
Respiratory system & 11.1 \\
Digestive system & 11.1 \\
Genital & 0 \\
Urinary tract & 11.1 \\
Musculoskeletal & 11.1 \\
Others & 55.6 \\
Chromosomal anomalies & 0 \\
\hline
\end{tabular}

Table 4 lists the average of length of stay of the nonaffected twin in the Neonatal Intensive Care Unit (NICU) according to the ICD-10.

A total of nine deaths of normal co-twins were observed during the study period. Table 5 shows the causes of death in normal twins according to the ICD-10 (WHO, 2015).

The nine deaths of healthy twins were associated with the following malformations according to the ICD-10: the 'Others' category accounted for five (55.6\%) deaths; Eyes, ears, face and circulatory system were responsible for 2 $(22.2 \%)$ deaths and $1(11.1 \%)$ death, respectively. A 10-fold higher risk of death in the normal twin was observed in cases of death of the anomalous twin $(p=.002$, OR 10.55, 95\% CI: 1.9-58.52).

The 'others' category of the ICD-10 was also associated with higher rates of prematurity, with a mean gestational age at birth of 27.9 weeks. Notably, delivery before 30.4 weeks was a significant predictor of fetal death $(p=.01)$.

\section{Discussion}

The present study investigated perinatal outcomes of twin gestations complicated by a discordant malformation. Monozygotic pregnancies are not always associated with malformations in both fetuses. This happens because $\mathrm{MZ}$ twins are not necessarily phenotypically identical. Early post-twinning mutational events can be responsible for this discordance.

The digestive system was found to be the most commonly affected system in our population studies, which differs from the other studies that indicated the circulatory system to be most commonly affected. This could be attributed to the sample populations studied (Chang et al., 2004; Kang et al., 2014).

Normal adverse outcomes for twins, such as death, small for gestational age, periventricular-intraventricular hemorrhage, use of surfactant, and mechanical ventilation had a lower gestational age of 33 weeks.

Other system changes were responsible for the highest average length of hospital stay in the neonatal ICU, compared to the healthy twin. The severity of hydrops fetalis explained pregnancies bearing this condition and fetuses with intrauterine growth restriction.

Single fetal death in a twin pregnancy is traditionally known as a serious complication of the entire pregnancy (Chang et al., 2004). Previous studies demonstrated the association between perinatal mortality and severe malformations (Heydanus et al., 1993; Kang et al., 2014; Nassar et al., 2000). Our study demonstrated a strong association between death of the normal twin and anomalous twin death. A total of nine deaths of the normal co-twin were observed during study period.

A systematic review of the literature to assess the risk of co-twin death after intrauterine death of one fetus revealed that the risks of $\mathrm{MC}$ and DC co-twin deaths were $12 \%$ (95\% CI: 7-18; $p=.02$ ) and 4\% (95\% CI: $2-7 ; p=.74)$, respectively (Ong et al., 2006).

Another recent systematic review of the literature evaluated the effect of SF versus conservative management on perinatal outcome of the normal fetus in DC and MC twins discordant for anencephaly. This review concluded that SF did not reduce perinatal mortality, but it resulted in significantly longer gestations and higher birth weight. Therefore, SF appears to be the management of choice in DC twins discordant for anencephaly. SF also increased birth weight in MC twins, but no clear recommendations were made in this review because of the complexity of this group (Lust et al., 2008).

The mean gestational age at delivery in the present study was 34.9 weeks. Previous studies reported that the mean gestational age at delivery in twin pregnancies with a fetal abnormality varied from 32 to 35.7 weeks (Chang et al., 2004; Gul et al., 2005; Harper et al., 2012; Malone et al., 1996). The presence of fetal hydrops was related to a 
preterm delivery mean of 27.9 weeks, which is approximately 4.1 weeks earlier than reported by Kang et al. (2014).

In conclusion, the management of twin pregnancies with one malformed fetus is complex. The first step is to distinguish the chronicity, because different management options are available for MC and DC twin pregnancies. Severe abnormalities should also be differentiated. The presence of a fetus with one major fetal malformation in a DC twin gestation increases the risk of preterm delivery (Gedikbasi et al., 2010). Although active management of DC twins improved gestational age and birth weight, these improvements are not statistically significant (Gedikbasi et al., 2010). Whether the best management is expectant or intervention in the case of MC twins is not certain. The preliminary results revealed no improvement in fetal outcome with active management. However, the number of cases in each group was too small to allow any definitive conclusions of the best approach to maximize the chances of survival of the normal co-twin and prevent preterm delivery. More prospective research is required to establish the best management of pregnancies with discordant anomalous twins.

\section{References}

American Congress of Obstetricians and Gynecologists (ACOG). (2004). ACOG practice bulletin 56: Multiple gestation: Complicated twin, triplet, and high-order multifetal pregnancy. Obstetrics \& Gynecology, 104, 869883.

Chang, Y. L., Chao, S. P., Cheng, P. J., Chung, C. L., Chueh, H. Y., Chanq, S. D., \& Spoonq, Y. K. (2004). Presence of a single fetal major anomaly in a twin pregnancy does not increase the preterm rate. Australian and New Zealand Journal of Obstetrics and Gynaecology, 44, 332-336.

Creasy, R. K., Resnik, R., \& Iams, J. D. (2009). Creasy and Resnik's maternal-fetal medicine principles and practice. Philadelphia, PA: Saunders/Elsevier.

DATASUS. (2015). Health information. Retrieved from DATASUS.http://tabnet.datasus.gov.br/cgi/tabcgi.exe? sinasc/cnv/nvuf.def.

Diana, W. B., Timothy, M. C., Mary, E. D., \& Fergal, D. M. (2010). Fetology, diagnosis and management of the fetal patient (2nd ed.). New York: McGraw Hill.

Gedikbasi, A., Akyol, A., Yildirim, G., Ekiz, A., Gul, A., \& Ceylan, Y. (2010). Twin pregnancies complicated by a single malformed fetus: Chorionicity, outcome and management. Twin Research and Human Genetics, 13, 501-507.

Gul, A., Cebeci, A., Aslan, H., Polat, I., Sozen, I., \& Ceylan, Y. (2005). Perinatal outcomes of twin pregnancies discordant for major fetal anomalies. Fetal Diagnosis and Therapy, 20, 244-248.

Harper, L. M., Odibo, A. O., Roehl, K. A., Longman, R. E., Macones, G. A., \& Cahill, A. G. (2012). Risk of preterm delivery and growth restriction in twins discordant for structural anomalies. American Journal of Obstetrics and Gynecology, 206, 1-5.
Heydanus, R., Santema, J. G., Stewart, P. A., Mulder, P. G., \& Wladimiroff, J. W. (1993). Preterm delivery rate and fetal outcome in structurally affected twin pregnancies: A retrospective matched control study. Prenatal Diagnosis, 13, 155-162.

Kang, H. J., Liao, A. W., Brizto, M. L., Francisco, R. P., Krebs, V. L., \& Zugaib, M. (2014). Prediction of intrauterine death and severe preterm delivery in twin pregnancies discordant for major fetal abnormality. European Journal of $\mathrm{Ob}$ stetrics \& Gynecology and Reproductive Biology, 175, 115118.

Little, J., \& Nevin, N. C. (1989a). Congenital anomalies in twins in Northern Ireland. III: Anomalies of the cardiovascular system, 1974-1978. Acta Genetica \& Medical Gemellology (Roma), 38, 27-35.

Little, J., \& Nevin, N. C. (1989b). Congenital anomalies in twins in Northern Ireland. II: Neural tube defects, 19741979. Acta Genetica \& Medical Gemellology (Roma), 38, 17-25.

Lust, A., De Catte, L., Lewi, L., Deprest, J., Loquet, P., \& Devlieger, R. (2008). Monochorionic and dichorionic twin pregnancies discordant for fetal anencephaly: A systematic review of prenatal management options. Prenatal Diagnosis, $28,275-279$.

Malone, F. D., Craigo, S. D., Chelmow, D., \& Alton, M. E. (1996). Outcome of twin gestations complicated by a single anomalous fetus. Obstetrics \& Gynecology, 88, 1-5.

Martin, J. A., Hamilton, B. E., \& Osteman, M. J. K. (2012). Three decades of twin births in the United States, 19802009. NCHS Data Brief, (80), 1-8.

Nassar, A. H., Adra, A. M., \& Gomez, O. (2000). Perinatal outcome of twin pregnancies with one structurally affected fetus: A case control study. Journal of Perinatology, 20, 8286.

Office for National Statistics. (2014). Births in England and wales by characteristics of birth birth 2: 2013 (statistical bulletin). London: Author.

Ong, S. S., Zamora, J., Khan, K. S., \& Kilby, M. D. (2006). Prognosis for the co-twin following single-twin death a systematic review. BJOG, 113, 992-998.

Purisch, S. E., DeFranco, E. A., Muglia, L. J., Odibo, A. O., \& Stamilio, D. M. (2008). Preterm birth in pregnancies complicated by major congenital malformations: A populationbased study. American Journal of Obstetrics and Gynecology, 199, 287.e1-e8.

Royal College of Obstetricians and Gynaecologists. (2008). 304 Green-top guideline: management of monochorionic twin 305 pregnancy; 51, pp. 1-13. https://www.rcog.org.uk/ en/guidelines-research-services/guidelines/gtg51/

Shek, N. W. M., Hillman, S. C., \& Kilby, M. D. (2014). Single-twin demise: Pregnancy outcome. Best Practice \& Research Clinical Obstetrics \& Gynaecology, 28, 249263.

WHO. (2015). International Statistical Classification of Diseases and Related Health Problems (ICD) Retrieved August 21, 2015. Retrieved from http://www.who. int/classifications/icd/en/. 